\title{
An SEIR Model with Infectious Latent and a Periodic Vaccination Strategy
}

\section{Islam A. Moneim}

Department of Scientific Computing, Faculty of Computers and Artificial intelligence, Benha University

Benha, PO 13518, Egypt

E-mail(corresp.): moneim97@yahoo.com

Received June 15, 2020; revised January 29, 2021; accepted February 16, 2021

\begin{abstract}
An SEIR epidemic model with a nonconstant vaccination strategy is studied. This SEIR model has two disease transmission rates $\beta_{1}$ and $\beta_{2}$ which imitate the fact that, for some infectious diseases, a latent person can pass the disease into a susceptible one. Here we study the spread of some childhood infectious diseases as good examples of diseases with infectious latent. We found that our SEIR model has a unique disease free solution (DFS). A lower bound $R_{0}^{\text {inf }}$ and an upper bound $R_{0}^{\text {sup }}$ of the basic reproductive number, $R_{0}$ are estimated. We show that, the DFS is globally asymptotically stable when $R_{0}^{\text {sup }}<1$ and unstable if $R_{0}^{\text {inf }}>1$. Computer simulations have been conducted to show that non trivial periodic solutions are possible. Moreover the impact of the contact rate between the latent and the susceptibles is simulated. Different periodic solutions with different periods including one, two and three years, are obtained. These results give a clearer view for the decision makers to know how and when they should take action against a possible new wave of these infectious diseases. This action is mainly, applying a suitable dose of vaccination just before a severe peak of infection occurs.
\end{abstract}

Keywords: modelling, simulation, disease free solution, two contact rates, global stability, periodic vaccination, $R_{0}$.

AMS Subject Classification: 34C60; 97M60; 37M05; 97M10.

\section{Introduction}

Some infectious diseases have a latent period, that is the time from being exposed to develop diseases symptoms. Infectious diseases, such as mumps, measles, AIDS, HBV and HCV are good examples of such diseases which have a latent period. Considering a latent period in modeling infectious diseases

Copyright (c) 2021 The Author(s). Published by Vilnius Gediminas Technical University

This is an Open Access article distributed under the terms of the Creative Commons Attribution License (http://creativecommons.org/licenses/by/4.0/), which permits unrestricted use, distribution, and reproduction in any medium, provided the original author and source are credited. 
leads the called SEIR epidemic models. Many researchers studied various kinds of SEIR models. For example, Moneim [19] studied the global behavior of the dynamics of the HBV disease. He considered an SEIR model with a constant vaccination rate and infectivity during the incubation period. Greenhalgh [4] and [6] considered SEIR and SEIRS models with density dependent contact rate. He used Hopf bifurcations to analyse these models. SEIRS models are studied in different ways by Zhang et al. [32] and Li and Muldowney [20] and Li et al. [12] introduced and analyzed an SEIRS model with time delay. Avila et al. [1] presented a work which focused in a family of SEIRS models with seasonality in the infection rate.

On the other hand some infectious diseases have an infective latent period such as mumps, measles, malaria and AIDS [11,31]. It means an individual from the latent class can transmit the disease into the susceptibles. So these kind of infectious diseases have two forces of infection in both of the infectious and latent periods. This fact has been modeled by some researchers and the mathematical analysis and results of these models have been published for examples, [11, 19, 31]. Models for HIV/AIDS with different infected stages have been studied. In these models there are two different infected stages with different ability of transmitting the diseases [24,25,28]. Also Samanta et al. [26] studied a delayed epidemic model with different constant contact rates.

Nowadays it is believed to be a fact that, some infectious diseases have periodicity in their dynamics amongst the population. These oscillations in the number of incidences are due to some circumstances such as, climate changes or opening and closing days in schools $[5,17]$. Remarkable peaks for influenza H1N1 are detected in many countries including Vietnam each year [2]. Shulgin et al. (1998) studied an SIR model with a vaccination rate $r(t)=p \delta(t-n T)$ where $p$ is a constant and $\delta(t)$ is the Dirac delta function. With this vaccination form, Dirac delta function, it has been found that, the disease free periodic solution is locally stable $[29,30]$. Jan and Xiao introduce a pulse vaccination strategy to study a dynamic model of dengue disease with periodic contact rates. They found that, the disease free periodic solution of the their impulsive system is globally asymptotically stable if $R_{0}<1$ and is unstable otherwise [7].

Two studies applied several simple and continuous time linear vaccinations based control strategies for a SEIR model which takes account the total population amounts as a refrain for the illness transmission. They found that under these vaccination strategies, the susceptibles, infected and infectious population tend asymptotically to zeros $[9,10]$. Moneim [16] considered an SIRS model, with a periodic vaccination rate, to study the spread of the influenza H1N1. He showed that, there is a periodic behavior due to the force of the periodic vaccination rate. Nonconstant periodic vaccination gives a good matching and a more realistic simulation of the periodic nature of some infectious diseases $[14,15,16]$. H1N1 has been investigated in many other studies like $[21,23,27]$ Here in this paper we assume that, the infection has a contagious latent period and the disease shows a fluctuation behavior in its propagation. Therefore, we consider an SEIR model with two disease transmission rates $\beta_{1}$ and $\beta_{2}$ and a time dependent periodic vaccination strategy $\rho(t)$ with period $T$. The periodic vaccination function $\rho(t)$ is imitating the seasonal variation 
in the reported cases for some infectious diseases due to climatological changes or opening and colsing dates of schools. We start off by formulating an SEIR model. Then obtaining a formula for the disease free state. To proceed further in analysing the model we need first to find out the key parameter, the so called, basic reproduction number $R_{0}$ which is the number of secondary cases generated by a single infective person contacted with the population at the disease free state. We prove that, the disease free solution (DFS) is globally asymptotically stable when $R_{0}<1$ and unstable if $R_{0}>1$. Finally a simulation results are conducted for our model with different types of vaccination functions and for estimated parameter values from the literature.

\section{The model}

We consider some assumptions to imitate the dynamics of an infectious disease with infective latent in present of applying a periodic vaccination strategy. These assumption are made as follows:

1. The population is mixed homogeneously and its total size is a constant $N$, which is split into four groups: susceptibles $S$, exposed (or latent) $E$, infected $I$ and finally recovered $R$.

2. As we assume that births balance deaths so, $\nu$ denotes the birth rate which is also the death rate.

3. The newborn individuals are vaccinated after birth at a constant rate $p$. This scheme is important as current vaccination strategy in the UK and many other countries, is to vaccinate as many children as near to birth as possible after the period of protection by maternal antibodies has lapsed. There is a nonconstant periodic vaccination strategy $\rho(t)$ with period $T$, which is designed to vaccinate susceptible peoples seasonally just before an expected outbreaks occur.

4. There are two infection rates $\beta_{1}$ and $\beta_{2}$. These rates are denoted as the potential contacts between a susceptible individual and an infected or latent one respectively. Potential contacts means that, contacts which produce a transmission of the infection.

5. Exposed persons transfer from the latent stage to join the infected class at a rate $\alpha$, therefore the latent period is $1 / \alpha$.

6. The infected population recovered by a constant rate $\gamma$, and $1 / \gamma$ is the infectious period.

Based on the previous assumptions, we consider the following SEIR model with a nonconstant periodic vaccination strategy to investigate the spread of some infectious diseases. Extending the works of $[15,17,19]$ our model can be represented as a non-linear system of four coupled ordinary differential equations as 
follows:

$$
\begin{aligned}
\frac{d S}{d t} & =\nu N(1-p)-\beta_{1} S I-\beta_{2} S E-(\nu+\rho(t)) S \\
\frac{d E}{d t} & =\beta_{1} S I+\beta_{2} S E-(\nu+\alpha) E \\
\frac{d I}{d t} & =\alpha E-(\nu+\gamma) I \\
\frac{d R}{d t} & =\nu N p+\rho(t) S+\gamma I-\nu R
\end{aligned}
$$

with $S+E+I+R=N$.

The vaccination rate $\rho(t)$ are non-zero, positive, continuous, non-constant periodic functions. The periodic vaccination function $\rho(t)$ is imitating the seasonal variation in the reported cases for some infectious diseases due to climatological changes or opening and colsing dates of schools. The system (2.1)-(2.4) does not have any equilibrium point but having a disease free solution (DFS) is still possible in the case that $E=I=0$.

\section{The disease free solution}

Here the vaccination rate $\rho(t)$ is a periodic function, therefore the system (2.1)(2.4) has no equilibrium point. On the other hand we expect that, there is a periodic disease free solution for Equations (2.1)-(2.4) when $E(t)=0$ and $I(t)=0$. In the case that, there is neither an infective nor a latent individual in the system, equation (2.1) is reduced to:

$$
\frac{d S}{d t}=\nu N(1-p)-(\nu+\rho(t)) S .
$$

The solution $\left(E^{*}, I^{*}\right)=(0,0)$ is an equilibrium for the exposed and infected individuals. Now, we shall find a solution for the susceptibles when $E(t)=0$ and $I(t)=0$. Also the stability of this solution will be investigated.

By integrating equation (3.1) we have,

$$
\begin{aligned}
& S(t)=S\left(t_{0}\right) \exp \left(-\nu\left(t-t_{0}\right)-\int_{t_{0}}^{t} \rho(\eta) d \eta\right)+N \nu(1-p) \\
& \quad \times \exp \left(-\nu\left(t-t_{0}\right)-\int_{t_{0}}^{t} \rho(\eta) d \eta\right) \int_{t_{0}}^{t} \exp \left(\nu\left(\eta-t_{0}\right)+\int_{t_{0}}^{t} \rho(\eta) d \eta\right) d \eta \\
& S\left(t_{0}+(n+1) T\right)=S\left(t_{0}+n T\right) \exp \left(-\nu T-\int_{t_{0}}^{t_{0}+T} \rho(\eta) d \eta\right)+N \nu(1-p) \\
& \quad \times \exp \left(-\nu T-\int_{t_{0}}^{t_{0}+T} \rho(\eta) d \eta\right) \int_{t_{0}}^{t_{0}+T} \exp \left(\nu\left(t-t_{0}\right)+\int_{t_{0}}^{t_{0}+T} \rho(\eta) d \eta\right) d \eta .
\end{aligned}
$$

Equation (3.2) is a recurrence relation between the susceptibles at times $t_{0}+n T$, for $n=1,2,3, \ldots$ Define a mapping $\Gamma$ such that $\Gamma\left(S_{n}\right)=S_{n+1}$ where $S_{n}=$ $S\left(t_{0}+n T\right)$. If $S_{n 1} \neq S_{n 2}$ we have that,

$$
\left|\Gamma\left(S_{n 1}\right)-\Gamma\left(S_{n 2}\right)\right| \leq\left|S_{n 1}-S_{n 2}\right| \exp (-\nu T) .
$$


Therefore, the mapping $\Gamma$ is a contraction mapping which has a unique fixed point $S^{*},[3,16]$. Moreover $S^{*}$, is depending on $t_{0}$, such that,

$$
\begin{aligned}
S^{*} & =S^{*} \exp \left(-\nu T-\int_{t_{0}}^{t_{0}+T} \rho(\eta) d \eta\right)+N \nu(1-p) \\
& \times \exp \left(-\nu T-\int_{t_{0}}^{t_{0}+T} \rho(\eta) d \eta\right) \int_{t_{0}}^{t_{0}+T} \exp \left(\nu\left(t-t_{0}\right)+\int_{t_{0}}^{t_{0}+T} \rho(\eta) d \eta\right) d \eta
\end{aligned}
$$

therefore,

$$
\begin{aligned}
& S^{*}\left(t_{0}\right)=\left(N \nu(1-p) \exp \left(-\nu T-\int_{t_{0}}^{t_{0}+T} \rho(\eta) d \eta\right)\right. \\
& \left.\times \int_{t_{0}}^{t_{0}+T} \exp \left(\nu\left(t-t_{0}\right)+\int_{t_{0}}^{t_{0}+T} \rho(\eta) d \eta\right) d \eta\right) \\
& \quad \times 1 / \exp \left(1-\nu T-\int_{t_{0}}^{t_{0}+T} \rho(\eta) d \eta\right) .
\end{aligned}
$$

Hence $S^{*}\left(t_{0}+T\right)=S^{*}(0)$. Therefor $S^{*}$ is a time dependent periodic function. Using $S^{*}=\hat{S}(t)$ and using an argument similar to $[16,17]$ we can easily deduce that $\hat{S}(t)=S^{*}(t+n T)$ for all $t \in[n T,(n+1) T]$ and $n \geq 0$, and $\hat{S}(t)$ is a periodic solution of period $T$ and it is the only disease free solution of $(2.1)-(2.4)$.

\section{The basic reproduction number}

It is well documented that, the basic reproduction number is the average expected number of the secondary cases produced by a single infected person entering the population when the population is in disease free situation. For our model there are two contact rates and in this case the average value of the expected number of secondary cases is given by,

$$
R_{0}=\frac{\beta_{2}(\nu+\gamma)+\alpha \beta_{1}}{(\nu+\gamma)(\nu+\alpha) T} \int_{0}^{T} \hat{S}(\eta) d \eta
$$

Definition 1. Define $R_{0}^{\text {sup }}$ as an upper bound and $R_{0}^{\text {inf }}$ a lower one for the value $R_{0}$ respectively where,

$$
R_{0}^{\text {sup }}=\frac{\beta_{2}(\nu+\gamma)+\alpha \beta_{1}}{(\nu+\gamma)(\nu+\alpha)} \sup _{t \in[0, T]} \int_{0}^{T} \frac{(\nu+\alpha)(\hat{S}(t-\phi) \exp [-(\nu+\alpha) \phi] d \phi}{1-\exp [-(\nu+\alpha) T]}
$$

and

$$
R_{0}^{i n f}=\frac{\beta_{2}(\nu+\gamma)+\alpha \beta_{1}}{(\nu+\gamma)(\nu+\alpha)} \inf _{t \in[0, T]} \int_{0}^{T} \frac{(\nu+\alpha)(\hat{S}(t-\phi) \exp [-(\nu+\alpha) \phi] d \phi}{1-\exp [-(\nu+\alpha) T]}
$$

Using a very similar proof to the corresponding one in [17] we can easily show that, $R_{0}^{\text {inf }} \leq R_{0} \leq R_{0}^{\text {sup }}$. 


\section{Stability of the DFS}

We start off by investigating the stability of periodic DFS $(\hat{S}(t), 0,0, \hat{R}(t))$. In this section we extend the results obtained by $[16,17]$ to our model in the case that there are two different contact rates. Using a similar argument to $[16,17]$ we try first to show that if $R_{0}^{\text {sup }}<1$ the DFS $(\hat{S}(t), 0,0, \hat{R}(t))$ is globally asymptotically stable and in the case that, $R_{0}^{\text {inf }}>1$ the DFS is unstable.

\subsection{Stability of the DFS when $R_{0}^{\text {sup }}<1$}

The first target is to show that, the DFS is globally asymptotically stable when $R_{0}^{\text {sup }}<1$. In this case we use an argument similar to that used in the proof of Theorem 1 [17].

\section{Lemma 1.}

$$
\limsup _{t \rightarrow \infty}(S-\hat{S})(t) \leq 0
$$

Proof. From Equation (2.1)

$$
\frac{d S}{d t}=\nu N(1-p)-\beta_{1} S I-\beta_{2} S E-(\nu+\rho(t)) S \leq \nu N(1-p)-(\nu+\rho(t)) S .
$$

As $(\hat{S}(t), 0,0, \hat{R}(t))$ is a solution of Equations $(2.1)-(2.4)$ when $I(t)=0$ and $E(t)=0$ then we have,

$$
\frac{d \hat{S}}{d t}=\nu N(1-p)-\beta_{1} \hat{S} \hat{I}-\beta_{2} \hat{S} \hat{E}-(\nu+\rho(t)) \hat{S}=\nu N(1-p)-(\nu+\rho(t)) \hat{S}
$$

Therefore $\frac{d(S-\hat{S})}{d t} \leq-(\nu+\rho(t))(S-\hat{S})$. By integrating this inequality we have that,

$$
(S-\hat{S})(t) \leq(S-\hat{S})\left(t_{0}\right) \exp \left[-\nu\left(t-t_{0}\right)-\int_{t_{0}}^{t} \rho(\eta) d \eta\right]
$$

Using the same argument as in Lemma 1 in [5] we deduce that, $\lim \sup _{t \rightarrow \infty}(S-$ $\hat{S})(t) \leq 0$.

Global stability of the disease free solution when $R_{0}^{\text {sup }}<1$, is the first main result in this section. Theorem 1 proves that, DFS is globally asymptotically stable (GAS) when $R_{0}^{\text {sup }}<1$. We give a proof of Theorem 1 by using a similar argument to Theorem 1 in [17].

Theorem 1. The DFS, $(\hat{S}, 0,0, \hat{R})$ is $G A S$ if $R_{0}^{\text {sup }}<1$,

Proof. As $\frac{d I}{d t}=\alpha E-(\nu+\gamma) I$, arguing similar to $[16,19]$ we can easily find that

$$
I^{\infty} \leq \alpha E^{\infty} /(\nu+\gamma)
$$

Here our idea of this proof is simple as that, Lemma 1 tells us that, given $\epsilon>0$ there exists $t_{3}$ such that $S(t) \leq \hat{S}(t)+\epsilon$ and $I \leq I^{\infty}+\epsilon$ for all $t \geq t_{3}$. Using 
Equation (2.2), $E(t)$ can be bounded above and by using this upper bound we can show that $E^{\infty}=0$. Now similar to Theorem $1[16,17]$ again suppose that $E^{\infty}>0$. By integrating Equation (2.2) we have that for $t \geq t_{0}>t_{3}$,

$$
\begin{aligned}
E(t) \leq & E\left(t_{0}\right) \exp \left[-(\nu+\alpha)\left(t-t_{0}\right)+\left[\beta_{2} E^{\infty}+\beta_{1} I^{\infty}+\epsilon\right)\right] \\
& \exp [-(\nu+\alpha) t] \int_{t_{0}}^{t}(\hat{S}(\eta)+\epsilon) \exp [(\nu+\alpha) \eta] d \eta
\end{aligned}
$$

Now

$$
\begin{aligned}
\exp [- & (\nu+\alpha) t] \int_{t_{0}}^{t} \hat{S}(\eta) \exp [(\nu+\alpha) \eta] d \eta=\int_{t_{0}}^{t} \hat{S}(\eta) \exp [-(\nu+\alpha)(t-\eta)] d \eta \\
& =\int_{0}^{t-t_{0}} \hat{S}(t-\phi) \exp [-(\nu+\alpha) \phi] d \phi .
\end{aligned}
$$

Suppose that $(k+1) T \geq t-t_{0} \geq k T$, therefore, we have

$$
\begin{aligned}
& \int_{0}^{t-t_{0}} \hat{S}(t-\phi) \exp [-(\nu+\alpha) \phi] d \phi=\int_{0}^{T} \hat{S}(t-\phi) \exp [-(\nu+\alpha) \phi] d \phi \\
& \quad \times(1+\exp [-(\nu+\alpha) T]+\exp [-(\nu+\alpha) 2 T]+\ldots+\exp [-(\nu+\alpha)(k-1) T]) \\
& \quad+\int_{k T}^{t-t_{0}} \hat{S}(t-\phi) \exp [-(\nu+\alpha) \phi] d \phi \leq \int_{0}^{T} \hat{S}(t-\phi) \exp [-(\nu+\alpha) \phi] d \phi \\
& \quad \times(1+\exp [-(\nu+\alpha) T]+\exp [-(\nu+\alpha) 2 T]+\ldots+\exp [-(\nu+\alpha) k T]), \\
& <\int_{0}^{T} \frac{\hat{S}(t-\phi) \exp [-(\nu+\alpha) \phi] d \phi}{1-\exp [-(\nu+\alpha) T]} .
\end{aligned}
$$

Therefore, for $t \geq t_{0}$ from Equation (5.1) we find that

$$
\begin{aligned}
E(t)< & E\left(t_{0}\right) \exp \left[-(\nu+\alpha)\left(t-t_{0}\right)\right] \\
& \left.+\left[\beta_{2} E^{\infty}+\beta_{1} I^{\infty}+\epsilon\right)\right] \int_{0}^{T} \frac{\hat{S}(t-\phi) \exp [-(\nu+\alpha) \phi] d \phi}{1-\exp [-(\nu+\alpha) T]} \\
\leq & N \exp \left[-(\nu+\alpha)\left(t-t_{0}\right)\right]+\left[\epsilon+E^{\infty} \frac{\beta_{2}(\nu+\gamma)+\alpha \beta_{1}}{(\nu+\gamma)(\nu+\alpha)}\right] \\
& \left(\sup _{t \in[0, T]} \int_{0}^{T} \frac{(\nu+\alpha) \hat{S}(t-\phi) \exp [-(\nu+\alpha) \phi] d \phi}{1-\exp [-(\nu+\alpha) T]}+\epsilon\right) .
\end{aligned}
$$

Choose $t_{4}>t_{0}$ large enough so that, $N \exp \left[-(\nu+\alpha)\left(t-t_{0}\right)\right]<\epsilon$, for $t \geq t_{4}$, then for $t \geq t_{4}$,

$$
\begin{aligned}
& E(t) \leq R_{0}^{s u p} E^{\infty} \\
& +\epsilon\left(1+\sup _{t \in[0, T]} \int_{0}^{T} \frac{(\nu+\alpha) \hat{S}(t-\phi) \exp [-(\nu+\alpha) \phi] d \phi}{1-\exp [-(\nu+\alpha) T]}+\frac{\beta_{2}(\nu+\gamma)+\alpha \beta_{1}}{(\nu+\gamma)(\nu+\alpha)} \epsilon\right) .
\end{aligned}
$$

Then choose $\epsilon$ small enough so that

$$
\epsilon\left(1+\sup _{t \in[0, T]} \int_{0}^{T} \frac{\hat{S}(t-\phi) \exp [-(\nu+\alpha) \phi] d \phi}{1-\exp [-(\nu+\alpha) T]}+\frac{\beta_{2}(\nu+\gamma)+\alpha \beta_{1}}{(\nu+\gamma)(\nu+\alpha)} \epsilon\right)<\psi E^{\infty},
$$


where $R_{0}^{\text {sup }}+\psi<1$ and $\psi>0$. Thus for $t \geq t_{4}$ we have that $E(t) \leq\left(R_{0}^{\text {sup }}+\right.$ $\psi) E^{\infty}$. Therefor $0 \leq E^{\infty} \leq\left(R_{0}^{\text {sup }}+\psi\right) E^{\infty}$. From this we have that $E^{\infty}=0$ and $I^{\infty}=0$. Hence $E(t) \rightarrow 0$ and $I(t) \rightarrow 0$ as $t \rightarrow \infty$.

To complete the proof it remains to prove that $S(t) \rightarrow \hat{S}(t)$ and $R(t) \rightarrow \hat{R}$ as $t \rightarrow 0$. As $\hat{R}(t)$ is a solution of Equation (2.4), when $I(t)=0$ we find that,

$$
\frac{d(R-\hat{R})}{d t}=\rho(t)(S-\hat{S})+\gamma I-\nu(R-\hat{R}) .
$$

As $E(t) \rightarrow 0$ and $I(t) \rightarrow 0$ as $t \rightarrow \infty$ there exists $t_{5}$ so that $E(t) \leq \epsilon_{1}$ and $I(t) \leq \epsilon_{1}$ for $t \geq t_{5}$ and using Lemma 1 we know that given $\epsilon_{1}>0$ there exists $t_{6}$ such that $S(t) \leq \hat{S}+\epsilon_{1}$ for all $t \geq t_{6}$. So for $t \geq t_{7}=\max \left(t_{5}, t_{6}\right)$ we have that,

$$
\frac{d(R-\hat{R})}{d t} \leq \rho(t) \epsilon_{1}+\gamma \epsilon_{1}-\nu(R-\hat{R}) \leq\left(\rho_{\max }+\gamma\right) \epsilon_{1}-\nu(R-\hat{R}) .
$$

Integrating this last inequality we have,

$$
\begin{aligned}
& (R-\hat{R})(t) \leq(R-\hat{R})\left(t_{7}\right) \exp \left[-\nu\left(t-t_{7}\right)\right]+\epsilon_{1}\left(\frac{\rho_{\max }+\gamma}{\nu}\right) \\
& \quad \times\left(1-\exp \left[-\nu\left(t-t_{7}\right)\right]\right) \leq N \exp \left[-\nu\left(t-t_{7}\right)\right]+\epsilon_{1}\left(\frac{\rho_{\max }+\gamma}{\nu}\right) .
\end{aligned}
$$

Now given $\epsilon_{2}>0$ choose $\epsilon_{1}>0$ such that $\epsilon_{1}\left(\left(\rho_{\max }+\gamma\right) / \nu\right)<\left(\epsilon_{2} / 2\right)$. Choose $t_{8}>t_{7}$ so that $N \exp \left[-\nu\left(t-t_{7}\right)\right] \leq\left(\epsilon_{2} / 2\right)$ for $t \geq t_{8}$. Hence given $\epsilon_{2}>0$ there is a time $t_{8}$ so that $(R-\hat{R})(t) \leq \epsilon_{2}$ for all $t \geq t_{8}$. As $S(t)=N-I(t)-R(t)-E(t)$ then $S(t) \geq N-\hat{R}(t)-\epsilon_{2}-2 \epsilon_{1}$ for $t \geq t_{8}$. Hence given $\epsilon \geq 0$, choose $\epsilon \geq$ $\left(\epsilon_{2}+2 \epsilon_{1}\right)$ then there exists $t_{9}$ so that $\bar{S}(t) \geq \hat{S}(t)-\epsilon$ for all $t \geq t_{9} \geq t_{8}$. But we have $S(t) \leq \hat{S}(t)+\epsilon$ for all $t \geq t_{6}$. therefore given $\epsilon \geq 0$ there exists $t_{9}$ such that $|S(t)-\hat{S}(t)| \leq \epsilon$ for all $t \geq t_{9}$. So $S(t) \rightarrow \hat{S}$ as $t \rightarrow \infty$. As $R(t)=N-S(t)-I(t)-E(t)$ thus we must have $R(t) \rightarrow \hat{R}(t)$ as $t \rightarrow \infty$, thus the proof of Theorem is completed.

Now recall that $R_{0}^{i n f}$ is a lower bound for our conjectured $R_{0}$. The next step is to study the stability of the DFS in the case that, $R_{0}^{i n f}>1$. Here we use an argument consisting of a mixture of those used in $[13,16,19]$. We can show that if $R_{0}^{i n f}>1$ the (DFS) is not stable and the disease will take off.

\subsection{Instability of the DFS when $R_{0}^{i n f}>1$}

In this subsection we shall study the stability of (DFS) when $R_{0}^{\text {inf }}>1$. We start off by considering that, if the infection is not present initially so that, $I(0)=E(0)=0$ and $S+R=N$. Applying the same argument as the proof of Theorem 1 we can easily find that, $(S(t), R(t)) \rightarrow(\hat{S}, \hat{R})$ as $t \rightarrow \infty$. Thus, the system (2.1)-(2.4) tends to the DFS as $t \rightarrow \infty$ if $E(0)=I(0)=0$ whatever the value of $R_{0}$. Therefore, without loss of generality we assume that, both $E(0)>0$ and $I(0)>0[16,17]$.

Recalling Definition 1 so, from equation (4.1) we have that:

$$
R_{0}^{i n f}=\frac{\beta_{2}(\nu+\gamma)+\alpha \beta_{1}}{(\nu+\gamma)(\nu+\alpha)} \inf _{t \in[0, T]} \int_{0}^{T} \frac{(\nu+\alpha) \hat{S}(t-\phi) \exp [-(\nu+\alpha) \phi]}{1-\exp [-(\nu+\alpha) T]} d \phi
$$


Theorem 2. If $R_{0}^{\text {inf }}>1$, then the DFS is unstable.

Proof. Suppose that the DFS is stable when $R_{0}^{\text {inf }}>1$. So if $(S, E, I, R)$ starts sufficiently close to $\left(S^{*}, 0,0, R^{*}\right)$ we must have that $(S, E, I, R) \rightarrow\left(S^{*}, 0,0, R^{*}\right)$ as $t \rightarrow \infty$. Then given $\epsilon=(1 / 2)\left(1-\left(1 / R_{0}^{\text {inf }}\right)\right)>0$ there exists $t_{1}>0$ such that $\left|S(t)-S^{*}\right| \leq \epsilon S^{*},|E(t)-0| \leq \epsilon,|I(t)-0| \leq \epsilon$ and $\left|R(t)-R^{*}\right| \leq \epsilon$ for all $t \geq t_{1}$. Pick $\epsilon_{1}>0$ so that $\left(R_{0}^{\text {inf }}+1\right)\left(1-\epsilon_{1}\right)>2$. Therefore, by Definition 1 Equation (4.1), there exists an integer $k$ such that

$$
\begin{aligned}
& \frac{\beta_{2}(\nu+\gamma)+\alpha \beta_{1}}{(\nu+\gamma)(\nu+\alpha)} \inf _{t \in[0, T]} \int_{0}^{T}(\nu+\alpha) \hat{S}(t-\phi) \exp [-(\nu+\alpha) \phi] d \phi \\
& (1+\exp [-(\nu+\alpha) T]+\exp [-(\nu+\alpha) 2 T]+\ldots+\exp [-(\nu+\alpha)(k-1) T]) \\
& >R_{0}^{\text {inf }}\left(1-\epsilon_{1}\right) .
\end{aligned}
$$

Choose $t_{0} \geq t_{1}: E\left(t_{0}\right) \geq E(0) \exp \left[-(\nu+\alpha) t_{0}\right]>0$ and $I\left(t_{0}\right) \geq I(0) \exp [-(\nu+$ $\left.\gamma) t_{0}\right]>0$ as $E(0)>0$ and $I(0)>0$, then choose $\epsilon_{2}$ such that:

$$
0<\epsilon_{2}<\min \left\{\frac{1}{2} I\left(t_{0}\right) \exp [-(\nu+\gamma) k T], \frac{\alpha}{2(\nu+\gamma)} E\left(t_{0}\right) \exp [-(\nu+\alpha) k T]\right\} .
$$

Also define

$$
\eta=\inf \left\{\phi \geq 0: I\left(t_{0}+\xi\right) \geq \epsilon_{2} \text { and } E\left(t_{0}+\xi\right) \geq \frac{\nu+\gamma}{\alpha} \epsilon_{2}, \text { for } \xi \in[0, \phi)\right\} .
$$

By continuity $\eta>0$ and if $\eta<\infty$ either $I\left(t_{0}+\eta\right)=\epsilon_{2}$ or $E\left(t_{0}+\eta\right)=$ $((\nu+\gamma) / \alpha) \epsilon_{2}$. This leads to a contradiction. From Equation (2.3) and by the definition of $\epsilon_{2}$ we find that,

$$
\begin{aligned}
I\left(t_{0}+\eta\right)= & I\left(t_{0}\right) \exp [-(\nu+\gamma) \eta] \\
& +\exp \left[-(\nu+\gamma)\left(t_{0}+\eta\right)\right] \int_{t_{0}}^{t_{0}+\eta} \alpha E(\chi) \exp [(\nu+\gamma) \chi] d \chi \\
\geq & I\left(t_{0}\right) \exp [-(\nu+\gamma) \eta]+\epsilon_{2}(1-\exp [-(\nu+\gamma) \eta])>\epsilon_{2},
\end{aligned}
$$

but on the other hand from Equation (2.2) we find that

$$
\begin{aligned}
& E\left(t_{0}+\eta\right) \geq E\left(t_{0}\right) \exp [-(\nu+\alpha) \eta]+\exp \left[-(\nu+\alpha)\left(t_{0}+\eta\right)\right] \\
& \quad \times \int_{t_{0}}^{t_{0}+\eta} \beta_{1} S(\chi) I(\chi) \exp [(\nu+\alpha) \chi] d \chi \geq E\left(t_{0}\right) \exp [-(\nu+\alpha) \eta] \\
& \quad+\exp \left[-(\nu+\alpha)\left(t_{0}+\eta\right)\right] \beta_{1} \epsilon_{2}(1-\epsilon) \int_{t_{0}}^{t_{0}+\eta} \hat{S}(\chi) \exp [(\nu+\alpha) \chi] d \chi
\end{aligned}
$$

Now

$$
\begin{aligned}
& \exp \left[-(\nu+\alpha)\left(t_{0}+\eta\right)\right] \int_{t_{0}}^{t_{0}+\eta} \hat{S}(\chi) \exp [(\nu+\alpha) \chi] d \chi \\
& =\int_{t_{0}}^{t_{0}+\eta} \hat{S}(\chi) \exp \left[-(\nu+\alpha)\left(t_{0}+\eta-\chi\right)\right] d \chi=\int_{0}^{\eta} \hat{S}\left(t_{0}+\eta-\phi\right) \exp [-(\nu+\alpha) \phi] d \phi
\end{aligned}
$$


Now if $\eta \leq k T$ then

$$
E\left(t_{0}+\eta\right) \geq E\left(t_{0}\right) \exp [-(\nu+\alpha) k T]>\frac{(\nu+\gamma)}{\alpha} \epsilon_{2}
$$

If $\eta \geq k T$ then

$$
\begin{aligned}
& \int_{0}^{\eta} \hat{S}\left(t_{0}+\eta-\phi\right) \exp [-(\nu+\text { alpha }) \phi] d \phi=\int_{0}^{T} \hat{S}\left(t_{0}+\eta-\phi\right) \exp [-(\nu+\alpha) \phi] d \phi \\
& \quad \times(1+\exp [-(\nu+\alpha) T]+\exp [-(\nu+\alpha) 2 T]+\ldots+\exp [-(\nu+\alpha)(k-1) T]) \\
& \quad+\int_{k T}^{\eta} \exp [-(\nu+\alpha) \phi] d \phi \geq \inf _{t \in[0, T]} \int_{0}^{T} \hat{S}(t-\phi) \exp [-(\nu+\alpha) \phi] d \phi \\
& \times(1+\exp [-(\nu+\alpha) T]+\exp [-(\nu+\alpha) 2 T]+\ldots+\exp [-(\nu+\alpha)(k-1) T]) \\
& \quad>\frac{\nu+\gamma}{\beta_{2}(\nu+\gamma)+\alpha \beta_{1}} R_{0}^{\text {inf }}\left(1-\epsilon_{1}\right), \text { using }(5.2) .
\end{aligned}
$$

As $R_{0}^{\text {inf }}>1$ and $\epsilon=\left(\left(R_{0}^{\text {inf }}-1\right) / 2 R_{0}^{\text {inf }}\right)>0$ then using Eq. (5.3)

$$
\begin{aligned}
& E\left(t_{0}+\eta\right) \geq E\left(t_{0}\right) \exp [-(\nu+\alpha) \eta]+\beta_{1} \epsilon_{2} \frac{1}{2}\left(\frac{1}{R_{0}^{\text {inf }}}+1\right) \frac{\nu+\gamma}{\beta_{2}(\nu+\gamma)+\alpha \beta_{1}} \\
& \times R_{0}^{\text {inf }}\left(1-\epsilon_{1}\right)=E\left(t_{0}\right) \exp [-(\nu+\alpha) \eta]+\frac{\beta_{1}(\nu+\gamma)}{2\left(\beta_{2}(\nu+\gamma)+\alpha \beta_{1}\right)} \\
& \times \epsilon_{2}\left(R_{0}^{\text {inf }}+1\right)\left(1-\epsilon_{1}\right)>\frac{\beta_{1}(\nu+\gamma)}{\beta_{2}(\nu+\gamma)+\alpha \beta_{1}} \epsilon_{2}, \text { as }\left(R_{0}^{\text {inf }}+1\right)\left(1-\epsilon_{1}\right)>2 .
\end{aligned}
$$

Hence $\eta<\infty$ leads to a contradiction so $\eta=\infty$ and $I\left(t_{0}+\xi\right) \geq \epsilon_{2}$ and $E\left(t_{0}+\xi\right) \geq\left((\nu+\gamma) \epsilon_{2} / \alpha\right)$ for all $\xi \geq 0$. This contradicts the fact that the trajectory tends to the DFE. Hence the DFE cannot be stable for $R_{0}^{i n f}>1$, and the proof is completed.

We can summarise the results obtained in this stability analysis as follows: The disease free solution for our SEIR model represented by Equations (2.1)-(2.4) with a nonconstant periodic and continuous vaccination rate $\rho(t)$ in $[0, T]$, is globally asymptotically stable if $R_{0}^{\text {sup }}<1$ and not stable if $R_{0}^{\text {inf }}>1$. These results prove that, the periodic vaccination strategy $\rho(t)$ with forces the system $(2.1)-(2.4)$ to have a periodic (DFS)equals to period $n T$ years including $n=$ $1,2,3$, where $T$ is the period of $\rho(t)$. We deduce that,

$$
\sup _{t \in[0, T]} \int_{0}^{T} \frac{\hat{S}(t-\phi) \exp [-(\nu+\alpha) \phi] d \phi}{1-\exp [-(\nu+\alpha) T]}<\frac{\nu+\gamma}{\beta_{2}(\nu+\gamma)+\alpha \beta_{1}}
$$

is the sufficient condition to keep the (DFS) globally asymptotically stable.

The obtained results, for our model with periodic vaccination and two different contact rates, extend the corresponding results obtained by $[5,16,17,19]$. These results are original for an SEIR model considering a combination of an infective latent period and a periodic vaccination strategy. 


\section{Simulation results}

Now we look numerically at the behaviour of the system different infectious diseases with infective latent period. The software package XPPAUTO is used to solve our system of nonlinear ordinary differential Equations (2.1)-(2.4). We used parameter values for Mumps, Measles, Rubella and Chickenpox. These parameter sets are estimated from the literature $[8,13,14,15,18,22]$. These parameters are taken as, the population $N$ is given a fixed value 1,000,000 and the other parameters are given the values as the following:

1. The infectious period $\gamma^{-1}=: 9.13,3.65,11.17$ and 11.67 days for chickenpox, measles, mumps and rubella respectively.

2. The latent period $\alpha^{-1}=: 15.22,9.49,18.26$ and 10.65 days for the same four infectious diseases respectively.

On the other hand $\beta_{2}$ is given a half of the value of $\beta_{1}$, and $0.00113, \beta_{1}=$ $0.0018,0.00081$ and $0.0007 /$ year for chickenpox, measles, mumps and rubella respectively to ensure that $R_{0}>1[15,19]$.

Our main target in this simulation, is to clarify the effect of latent infectivity in the dynamics of the infectious diseases. We solve our model for different values of the infection rate of the latent population $\beta_{2}$. For parameter values of all the diseases under study, we found that, infected population is increasing monotonically when $\beta_{2}$ increases. We plot these solutions against a range of the parameter values of $\beta_{2}$.

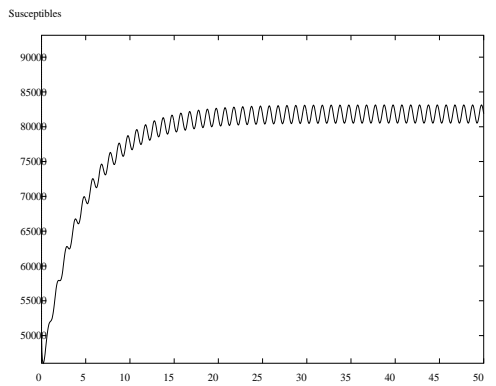

(a)

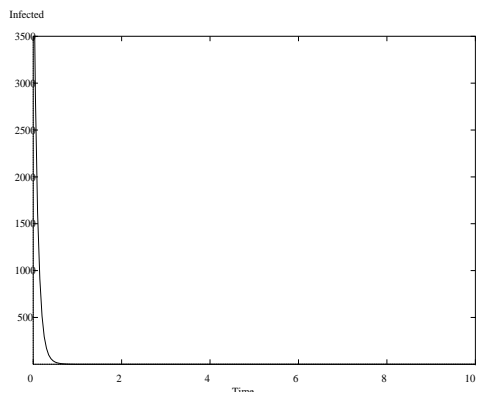

(b)

Figure 1. Plots the output results of $(a)$ the susceptibles $S(t)$ and $(b)$.

Also our simulations have been conducted for two different states first when $R_{0}<1$ and the second when $R_{0}>1$ and we found that, the vaccination of the disease has a threshold level $p_{c}$ depending on the values of the vaccination parameters $\rho(t)$ and the constant convectional vaccination one $p$. To keep the reproductive number $R_{0}$ under one in value the vaccination parameters should be large enough. When using the convectional constant strategy only the threshold vaccination level is $p_{c}=95 \%$. On the other hand if we use a periodic vaccination this level is reduced to be less than $60 \%[14,15]$. If the 
vaccination value is not sufficient to exceed the threshold value $p_{c}$, then $R_{0}$ stays above one in value and the disease becomes endemic. In the case that, $R_{0}>1$ periodic solutions of period one, two and three years are obtained and all of them appears to be stable by the end of the time range.

Figure $1(a)$ and $(b)$ shows that when the basic reproduction number is smaller than one in value, the susceptible population $S(t)$ is fluctuating and the infected $I(t)$ tends to zero. Now if $R_{0}<1$, both of $S(t)$ and $I(t)$ approach the disease free solution and the disease dies out. Here, Figure 1 shows that, the disease free solution is a periodic solution with period one year.

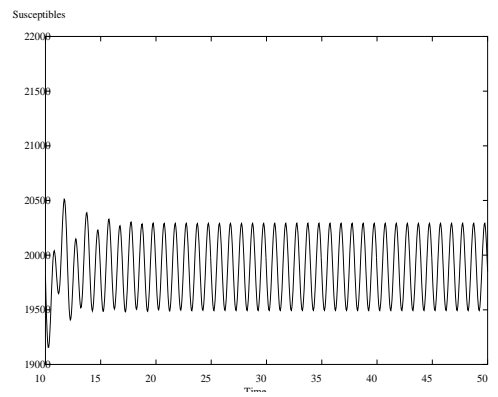

(a)

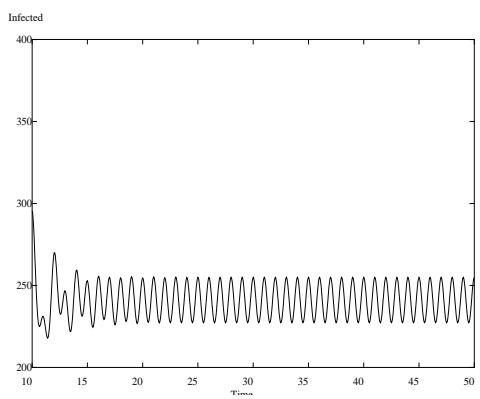

(b)

Figure 2. One year periodic solution of our model and plots approximate results of $(a)$ the susceptibles $S(t)$ and $(b)$ the infected $I(t)$ against time in years, when $\left(R_{0}>1\right)$.

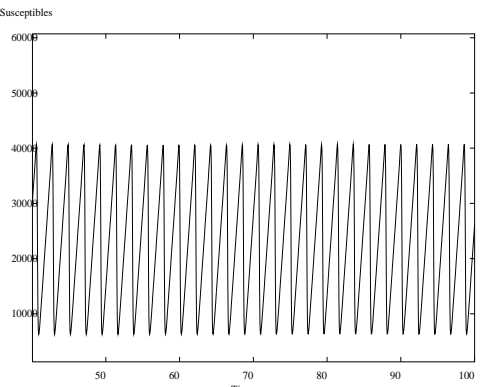

(a)

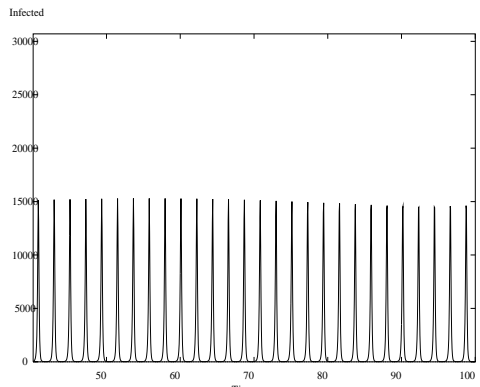

(b)

Figure 3. Two years periodic solution of our model and plots approximate results of $(a)$ the susceptibles $S(t)$ and $(b)$ the infected $I(t)$ against time in years, when $R_{0}>1$.

Figure $2(a)$ and $(b)$ shows that, there is a periodic solution with period one year of our model when $R_{0}>1$. Moreover, this solution is stable. Also Figures 3-4 give approximate solutions of our model when the reproduction number satisfies $R_{0}>1$. These figures show that, two and three years periodic stable solutions are obtained from our simulations and the disease persists in 
the population. Existence of periodic solutions with different periods means that epidemics can be occur repeatedly when $R_{0}>1$.

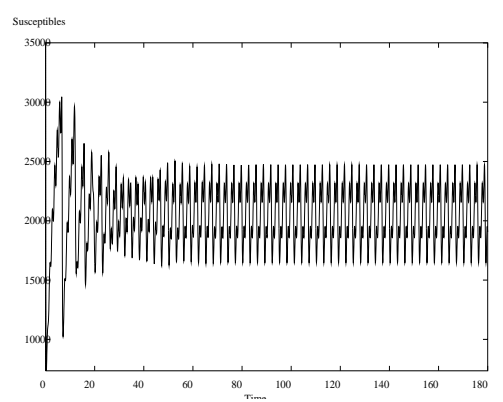

(a)

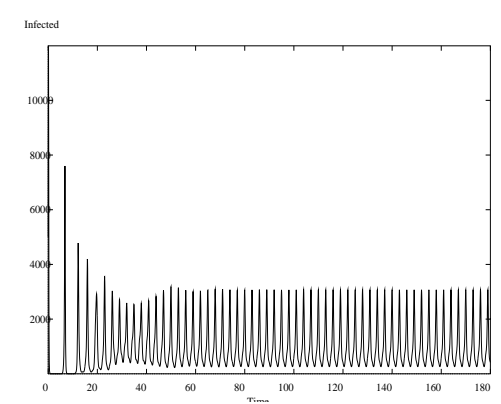

(b)

Figure 4. Three years periodic solution of our model and plots approximate results of $(a)$ the susceptibles $S(t)$ and $(b)$ the infected $I(t)$ against time in years, when $R_{0}>1$.

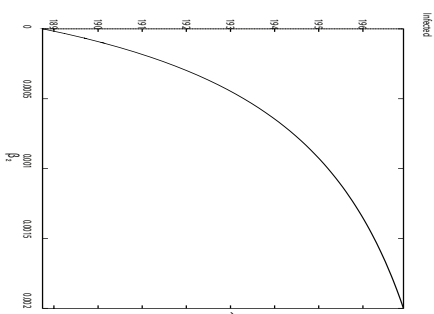

a)

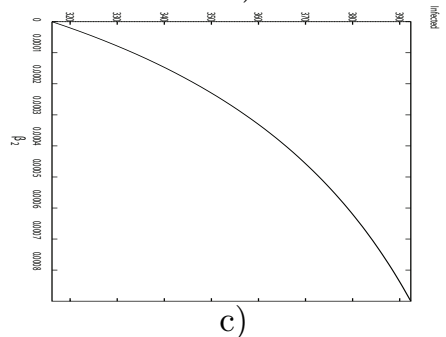

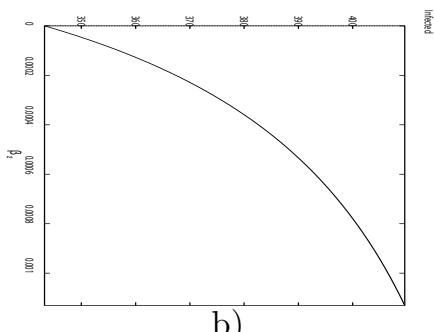

b)

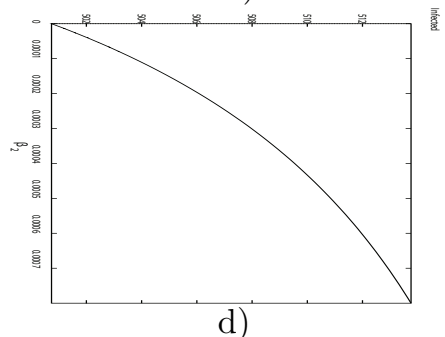

Figure 5. Plots the infected population $I(t)$ against the contact rate between latent and susceptibles $\beta_{2}$ and corresponding to parameter values of (a) measles, (b) mumps, (c) chickenpox and (d) rubella respectively.

Figures $5(a),(b),(c)$ and $(d)$ show that, there is a remarkable increase in the number of infected cases corresponding to the increase of the value of the contact rate between latent and susceptibles $\left(\beta_{2}\right)$. Figure 5 shows that the highest effect of the contact rate between latent and susceptibles $\beta_{2}$ is found in the chickenpox and mumps diagrams, while the lowest effect is observed in the measles diagram. As expected for all parameter sets of measles, mumps, chickenpox and rubella, there is a significant effect of the infectivity in the latent 
period and it is clearly confirmed from by the results plotted in Figure 5. This effect should be further considered in the future investigations.

\section{Conclusions}

In this paper we have studied an SEIR model with periodic nonconstant vaccination and two types of contact rate, one between infected and susceptibles and the other between latent and susceptibles. The main target of this paper is to study the effect of adding a realistic factor, which is the contact rate between the latent and the susceptibles. This idea will be studied using an SEIR model with nonconstant vaccination. We applied this idea for investigating the transmission of childhood infectious diseases. Some previous researches studied the study of the dynamics of infectious diseases with two contact rates in. However almost all of these studies didn't combine this idea with any kind of periodic vaccination.

This paper considered two transmission rates $\beta_{1}$ between infected and susceptibles and $\beta_{2}$ between latent and susceptibles. Also we use a vaccination rate, which is a nonconstant, positive, bounded and continuous periodic function of time, to vaccinate the susceptibles from all ages. $R_{0}$, the basic reproductive number, is the key parameter for our investigations in this study. We have conjectured a lower bound $R_{0}^{\text {inf }}$ and an upper bound $R_{0}^{\text {sup }}$ for our key parameter $R_{0}$. We have shown that with a non-constant vaccination rate $\rho(t)$, there is a unique DFS for the system (2.1)-(2.4). Moreover this solution is periodic with the same period of the vaccination function. Formula for $R_{0}$, when the vaccination function is $\rho(t)$ was also derived.

The stability of the DFS of our SEIR model with a general periodic vaccination rate was studied in the case that, there are two ways of passing the infection. We found that, when $R_{0}^{\text {sup }}<1$, the DFS is globally asymptotically stable. In this case the disease becomes extinct from the population. On the other hand if $R_{0}^{\text {inf }}>1$, the DFS becomes unstable and the disease will fire up and becomes endemic. The results of the stability analysis of the DFS lead to derive a condition under which the vaccination strategy could prevent outbreaks or severe epidemics. This sufficient condition is to keep $\hat{S}$ small enough so that Equation (5.4) holds. Our results for the SEIR model given by equations (2.1)-(2.4) were obtained when the vaccination strategy is a periodic function and our model has two disease transmission rates $\beta_{1}$ and $\beta_{2}$. Our results are original for this kind of SEIR model with two disease transmission rates and periodic vaccination.

The simulations conducted in this work for a model with an infectious latent and a periodic vaccination rate and for a set of four different childhood infectious diseases. The stability analysis of the (DFS) of our model has been confirmed. The results show that the DFS is stable when $R_{0} \leq 1$ and in this case the disease dies out. On the other hand if $R_{0}>1$ then the (DFS) is unstable and the diagrams show that there are stable periodic solutions with different periods including one, two and three years periodic solutions. As expected the periodic vaccination rate force the solutions to be periodic and there is no equilibrium point. 
The simulation results, also have indicated that introducing a new contact rate between latent and susceptibles generates significant changes in the number of infected persons. This result is obtained for each disease parameter set as shown in Figures $5(a)-(d)$. Moreover these diagrams show that, these changes in the levels the infected individuals, are increasing monotonically with the increase of $\beta_{2}$, the contact rate between the latent and the susceptibles. Finally the simulation results obtained and represented in Figures 1-5, give a clearer insight into the dynamics of these diseases in the case that, there is a chance of transmitting the disease during the latent period.

\section{References}

[1] E. Avila, E. Rivero and G. Emilio. Golobal dynamics of a periodic SEIRS model with general incidence rate. International Journal of Differential Equations, 2017:1-14, 2017. https://doi.org/10.1155/2017/5796958.

[2] M.F. Boni, B.H. Manh, P.Q. Thai and et al. Modelling the progression of pandemic influenza a $(\mathrm{H} 1 \mathrm{~N} 1)$ in Vietnam and the opportunities for reassortment with other influenza viruses. BMC Medicene, 7(43), 2009. https://doi.org/10.1186/1741-7015-7-43.

[3] T.A. Burton. Stability and periodic solutions of ordinary and functional differential equations. Academic Press, New York, 1985.

[4] D. Greenhalgh. Hopf bifurcation in epidemic models with a latent period and nonpermanent immunity. Math. Comput. Model, 25:85-93, 1997.

[5] D. Greenhalgh and I.A. Moneim. SIRS epidemic model and simulations using different types of seasonal contact rate. Systems Analysis Modelling Simulation, 43(5):573-600, 2003. https://doi.org/10.1080/023929021000008813.

[6] D. Grenhalgh. Some results for an SEIR epidemic model with density dependence in the death rate. IMA J. Math. Appl. Med. Biol., 9(2):67-106, 1992. https://doi.org/10.1093/imammb/9.2.67.

[7] R. Jan and Y. Xiao. Effect of pulse vaccination on dynamics of dengue with periodic transmission functions. Advances in Difference Equations, 219(1):368, 2019. https://doi.org/10.1186/s13662-019-2314-y.

[8] T. Kitano. Dynamic transmission model of routine mumps vaccination in Japan. Epidemiology and Infection, 147:1-8, 2018. https://doi.org/10.1017/S0950268818003230.

[9] M. De la Sen, A. Ibeas and S. Alonso-Quesada. On vaccination controls for the SEIR epidemic model. Communications in Nonlinear Science and Numerical Simulation, 17(6):3888-3904, 2012. https://doi.org/10.1016/j.cnsns.2011.10.012.

[10] M. De la Sen and S.Alonso-Quesada. Vaccination strategies based on feedback control techniques for a general SEIR epidemic model. Applied Mathematics and Computation, 218(7):2637-2658, 2011. https://doi.org/10.1016/j.amc.2011.09.036.

[11] G. Li and Z. Jin. Global stability of a SEIR epidemic model with infectious force in latent, infected and immune period. Chaos, Solitons and Fractals, 25(2):11771184, 2005. https://doi.org/10.1016/j.chaos.2004.11.062. 
[12] M.Y. Li, J.S. Muldoweney, L.C. Wang and J. Karsai. Global dynamics of an SEIR epidemic model with a varying total population size. Math. Biosci., 160(2):191213, 1999. https://doi.org/10.1016/S0025-5564(99)00030-9.

[13] I.A. Moneim. Seasonally varying epidemics with and without latent period: A comparative simulation study. IMA J. of Mathematical Medicine and Biology, 24(1):1-15, 2007. https://doi.org/10.1093/imammb/dq1023.

[14] I.A. Moneim. Different vaccination strategies for measles diseases: A simulation study. Journal of Informatics and Mathematical Sciences, 3:227-236, 2011.

[15] I.A. Moneim. Efficiency of different vaccination strategies for childhood diseases: A simulation study. Advances in Bioscience and Biotechnology, 4(2):193-205, 2013. https://doi.org/10.4236/abb.2013.42028.

[16] I.A. Moneim. Modeling and simulation of the spread of H1N1 flu with periodic vaccination. IJB., 9(1), 2016. https://doi.org/10.1142/S1793524516500030.

[17] I.A. Moneim and D. Greenhalgh. Threshold and stability results for an SIRS epidemic model with a general periodic vaccination strategy. Journal of biological systems, 13(2):131-150, 2005. https://doi.org/10.1142/S0218339005001446.

[18] I.A. Moneim and D. Greenhalgh. Use of a periodic vaccination strategy to control the spread of epidemics with seasonally varying contact rate. Mathematical Bioscience and Engineering, 13(2):591-611, 2005. https://doi.org/10.3934/mbe.2005.2.591.

[19] I.A. Moneim and H.A. Khalil. Modelling and simulation of the spread of HBV disease with infectious latent. A M., 6(5):745-753, 2015. https://doi.org/10.4236/am.2015.65070.

[20] M.Y.Li and J.S. Muldoweney. Global dynamics of a SEIR epidemic model with vertical transmission. SIAM J. Appl.Math., 62(1):58-69, 2001. https://doi.org/10.1137/S0036139999359860.

[21] H. Nishiur and H. Inaba. Estimation of the incubation period of influenza a (H1N1) among imported cases: Addressing censoring using outbreak data origin of importation. J Theor Biol., 272(1):123-130, 2011. https://doi.org/10.1016/j.jtbi.2010.12.017.

[22] O.J. Peter, O.A. Afolabi, A.A. Victor and et al. Mathematical model for the control of measles. J. Appl. Sci Environ. Manage, 22(4):571-576, 2018. https://doi.org/10.4314/jasem.v22i4.24.

[23] S. Rausanu and C. Grosan. A hierarchical network model for epidemic spreading. Analysis of a H1N1 virus spreading in Romania. Appl. Math Comput, 233:39-54, 2014. https://doi.org/10.1016/j.amc.2013.12.176.

[24] S. Saha and G.P. Samanta. Modelling and optimal control of HIV/AIDS prevention through PrEP and limited treatment. Physica A., 516:280-307, 2019. https://doi.org/10.1016/j.physa.2018.10.033.

[25] G.P. Samanta. Analysis of a nonautonomous HIV/AIDS epidemic model with distributed time delay. Mathematical Modelling and Analysis, 15(3):327-347, 2010. https://doi.org/10.3846/1392-6292.2010.15.327-347.

[26] G.P. Samanta, P. Sen and A. Maiti. A delayed epidemic model of diseases through droplet infection and direct contact with saturation incidence and pulse vaccination. Systems Science \& Control Engenering: An Open Access Journal, 4(1):320-333, 2016. https://doi.org/10.1080/21642583.2016.1246982. 
[27] M. Sasuzzoha, M. Singh and D. Lucy. Parameter estimation of influenza epidemic model. Appl. Math Comput, 220:616-629, 2013. https://doi.org/10.1016/j.amc.2013.07.040.

[28] S. Sharma and G.P. Samanta. Dynamical behaviour of an HIV/AIDS epidemic model. Differ Equ Dyn Syst., 22:369-395, 2014. https://doi.org/10.1007/s12591013-0173-7.

[29] B. Shulgin, L. Stone and Z. Agur. Pulse vaccination strategy in the SIR epidemic model. Bull. Math. Biol., 60:1123-1148, 1998.

[30] L. Stone, B. Shulgin and Z. Agur. Theoretical examination of the pulse vaccination policy in the SIR epidemic model. Math. Comput. Model., 31(4-5):207-215, 2000 .

[31] X.Li and B. Fang. Stability of an age-structured SEIR epidemic model with infectivity in latent period. Appl. Appl. Math, 4(1):218-236, 2009.

[32] J. Zhang and Z. Ma. Global stability of SEIR model with saturating contact rate. Math. Biosci., 185(1):15-32, 2003. https://doi.org/10.1016/S00255564(03)00087-7. 